\title{
A TOPOLOGICAL SPACE WITHOUT A COMPLETE QUASI-UNIFORMITY
}

\author{
HANS-PETER A. KUNZI ${ }^{1}$ AND PETER FLETCHER
}

\begin{abstract}
We show that an example of Burke and van Douwen has no complete quasi-uniformity. Moreover, we show that it is almost finitely-fully normal but not almost $\boldsymbol{R}_{0}$-fully normal.
\end{abstract}

0. Introduction. Every topological space admits a quasi-uniformity. The problem whether every topological space admits a complete quasi-uniformity is considered in [3, Problem C], where an example is given of a $T_{1}$-space that admits a complete, but no convergence complete, quasi-uniformity. In this note we show that a locally compact separable normal $M$-space of D. K. Burke and E. K. van Douwen admits no complete quasi-uniformity, thereby answering an old question in the theory of quasi-uniform spaces. Moreover, we show that this space is an almost finitely-fully normal countably paracompact space that is not almost $\boldsymbol{\aleph}_{0}$-fully normal. It is interesting to compare these results with the recent results of K. P. Hart [4] that M. E. Rudin's Dowker space is both orthocompact and finitely-fully normal; for it follows readily from Hart's results that, while Rudin's space is not almost $\boldsymbol{\aleph}_{0}$-fully normal, it does admit a complete quasi-uniformity.

1. Definitions and a lemma. A quasi-uniformity on a set $X$ is a filter $\mathcal{Q}$ on $X \times X$ such that (a) each member of $\mathcal{Q}$ is a reflexive relation on $X$, and (b) if $U \in \mathcal{Q}$ then $V \circ V \subset U$ for some $V \in \mathcal{Q}$. The pair ( $X, \mathcal{Q})$ is called a quasi-uniform space. A filter $\mathscr{F}$ on $(X, \mathcal{Q})$ is a Cauchy filter provided that for each $U \in \mathcal{U}$ there exists $p \in X$ so that $U(p) \in \mathscr{F}$, and $(X, \mathcal{Q})$ is said to be complete provided that every Cauchy filter has a cluster point. The topology $\tau(\mathcal{U})=\{G \subset X$ : for each $x \in G$ there is $U \in \mathcal{Q}$ with $U(x) \subset G\}$ is called the topology induced by $\mathcal{U}$. A topological space $(X, \tau)$ admits $\mathcal{Q}$ provided that $\tau$ is the topology induced by $\mathcal{Q}$. Let $(X, \tau)$ be a topological space and let $\mathscr{B}$ be the collection of reflexive transitive relations $V$ on $X$ for which $V(x) \in \tau$ for all $x \in X$. Then $\mathscr{B}$ is a filterbase for a quasi-uniformity $\mathcal{Q}$. Moreover, using the observation of W. J. Pervin [8] that for each open set $G, G \times G \cup(X \backslash G)$ $\times X \in \mathcal{Q}$, we see that $(X, \tau)$ admits $\mathcal{Q}$. It is known that this quasi-uniformity $\mathscr{Q}$ is complete if and only if every ultrafilter on $X$ without a cluster point has a closure-preserving subcollection without a cluster point [3, p. 59]. Consequently, a

Received by the editors February 14, 1983 and, in revised form, June 28, 1983.

1980 Mathematics Subject Classification. Primary 54E15, 54D20.

Key words and phrases. Almost $\boldsymbol{\aleph}_{0}$-fully normal, almost finitely-fully normal, complete quasi-uniformity.

'While working on this paper, the first author was supported by the Schweizerischer Nationalfonds. 
regular space that is either almost real-compact or weakly orthocompact admits a complete quasi-uniformity.

Throughout, if $\mathcal{R}$ is a cover of a space $X$, and $A$ is a subset of some member of $\mathscr{R}$, we say that $A$ is a refiner of $R$. A space $X$ is almost finitely-fully normal (almost $\boldsymbol{\aleph}_{0}$-fully normal) [6] provided that if $\varrho$ is an open cover of $X$ there is an open refinement $R$ of $\varrho$ with the property that if $M$ is a finite (countable) set and $M$ is a refiner of $\{\operatorname{st}(x, \mathcal{R}) \mid x \in X\}$, then $M$ is also a refiner of $\circlearrowright$.

We begin with a slight extension of a result of G. Aquaro [1] and K. Morita [7, Lemma 4.3].

Lemma 1. A space $X$ is almost finitely-fully normal (almost $\boldsymbol{\aleph}_{0}$-fully normal) if and only if $X$ is normal, and for each open cover $\circlearrowright$ of $X$ there is a locally finite open cover $R$ of $X$ so that if $M$ is a refiner of $R$ and $M$ is a finite (countable) set, then $M$ is a refiner of $\mathcal{C}$.

2. The example. The example $X$ under consideration is described completely in [2]. For our purposes it is enough to know the following details: The ground set is $\mu \cup(\omega \times \omega), \mu$ and $\omega$ considered disjoint, where $\mu$ is a regular cardinal, and there is a collection $F=\left\{f_{\alpha}: \alpha \in \mu\right\}$ such that

(0) for each $\alpha \in \mu, f_{\alpha} \in{ }^{\omega} \omega$,

(1) each $f_{\alpha}$ is nondecreasing,

(2) $f_{\alpha}<* f_{\beta}$ if $\alpha<\beta$,

(3) there is no $g \in{ }^{\omega} \omega$ so that $f_{\alpha} \leqslant{ }^{*} g$ for all $\alpha \in \mu$.

(As usual, we say $f<^{*} g$ provided that for all but finitely many $n \in \omega, f(n)<g(n)$.)

The points of $\omega \times \omega$ are isolated, and basic open sets about $\alpha \in \mu$ with $0 \leqslant \beta<\alpha$ $<\mu$ and $m \in \omega$ are of the form

$$
U(\alpha, \beta, m)=(\beta, \alpha] \cup\left\{\langle k, n\rangle: k \geqslant m \text { and } f_{\beta}(k)<n \leqslant f_{\alpha}(k)\right\} .
$$

If $\alpha \in \mu$ and $\alpha=0$, basic open sets about $\alpha$ are of the form

$$
U(0, \beta, m)=\{0\} \cup\left\{\langle k, n\rangle: k \geqslant m, n \leqslant f_{0}(k)\right\} \quad \text { where } m \in \omega .
$$

LEMma 2. If $S$ is a cofinal subset of $\mu$, then $A=\left\{k \in \omega:\left\langle f_{s}(k)\right\rangle_{s \in s}\right.$ is eventually bounded $\}$ is an initial segment of $\omega$.

Proof. Since each $f_{\alpha}$ is nondecreasing, we note that if $a<b<\omega$ and $b \in A$, then $a \in A$. Assume $A=\omega$. Then for all $n \in \omega$ there are $s_{n} \in S$ and $k_{n} \in \omega$ such that if $s \in S$ and $s>s_{n}$ then $f_{s}(n)<k_{n}$. Define $g: \omega \rightarrow \omega$ by $g(n)=k_{n}$. There is a tail $S^{\prime}$ of $S$ such that if $s \in S^{\prime}$ then $f_{s}(n)<g(n)$ for all $n \in \omega$. Let $f_{\alpha} \in F$. There is an $s \in S^{\prime}$ with $\alpha<s$; by (2), $f_{\alpha} \leqslant{ }^{*} f_{s}$, and thus $f_{\alpha} \leqslant g$. We have shown that (3) fails-a contradiction.

$X$ admits no complete quasi-uniformity. For each $x \in \mu$ and $m \in \omega$, set $F(x, m)=$ $\left\{\langle k, n\rangle: k \geqslant m\right.$ and $\left.f_{x}(k)<n\right\}$ and let $\mathscr{F}$ be the filter for which $\{F(x, m): x \in \mu$ and $m \in \omega\}$ is a filter base. Clearly no point of $\omega \times \omega$ is a cluster point of $\mathscr{F}$. If $\rho \in \mu$ and $m \in \omega$, then $U(\rho, 0,0) \cap F(\rho, m)=\varnothing$. Therefore $\mathscr{F}$ is a filter without a cluster point. We show that $\mathscr{F}$ is a Cauchy filter with respect to each quasi-uniformity that $X$ admits. Let $\mathfrak{V}$ be such a quasi-uniformity, let $V \in \mathcal{V}$ and let $W \in \mathscr{V}$ so $W^{2} \subset V$. For 
each $x \in \mu$ choose $\beta_{x} \in \mu$ and $m_{x} \in \omega$ so that $U\left(x, \beta_{x}, m_{x}\right) \subset W(x)$. By the Pressing-Down Lemma, there is a cofinal subset $S$ of $\mu, \beta \in \mu$ and $j \in \omega$ so that, for all $s \in S, U(s, \beta, j) \subset W(s)$. We note that $\beta<s$ for each $s \in S$. Set $A^{\prime}=\{k \in \omega$ : $\left\langle f_{s}(k)\right\rangle_{s \in S}$ is bounded $\}$. By Lemma $2, A^{\prime}$ is finite. There is $e \in \omega$ so that if $k \geqslant e$ then $\left\{f_{s}(k): s \in S\right\}$ is unbounded. Let $r=\max \{e, j\}$. For each $k \geqslant r$, define a function $a_{k}: \omega \rightarrow S$ by letting $a_{k}(n)$ be the least ordinal $\alpha \in S$ so $f_{\alpha}(k) \geqslant n$. Let $\gamma=\sup \left\{a_{k}(n): k \geqslant r, n \in \omega\right\}$; there exists $s_{0} \in S$ with $\gamma<s_{0}<\mu$. Let $k \geqslant r$ and let $m \in \omega$. Then $a_{k}(m) \in U\left(s_{0}, \beta, j\right) \subset W\left(s_{0}\right)$ so $W\left(a_{k}(m)\right) \subset W^{2}\left(s_{0}\right) \subset V\left(s_{0}\right)$. We show that $F(\beta, r) \subset V\left(s_{0}\right)$ so $V\left(s_{0}\right) \in \mathscr{F}$ as required. Let $\langle k, n\rangle \in F(\beta, r)$. Then $k \geqslant r \geqslant j$ and $f_{\beta}(k)<n$ so

$$
\langle k, n\rangle \in U\left(a_{k}(n), \beta, j\right) \subset W\left(a_{k}(n)\right) \subset V\left(s_{0}\right) .
$$

COROllary. The space $X$ is not weakly orthocompact. (Indeed, $X$ is a transitive space that is not preorthocompact; see [3, Lemma 6.16 and Corollary 5.11].)

For the nonce, mimicking terminology of E. Hewitt, we say a topological space is $q$-complete provided it admits a complete quasi-uniformity. In this terminology, Proposition 3.12 of [3] shows that the perfect preimage of a $q$-complete space is $q$-complete. The present example shows that this result does not obtain if perfect maps are replaced by quasi-perfect maps.

3. Further properties of the example. $X$ is almost finitely-fully normal. Let $\mathscr{B}$ be an open cover of $X$. Without loss of generality, we assume that for each $x \in \mu$ there is $B_{x} \in \mathscr{B}$ of the form $B_{x}=U\left(x, \beta_{x}, m_{x}\right)$ and that all remaining members of $\mathscr{B}$ are isolated points. Since $\mu$ is a regular cardinal, by the Pressing-Down Lemma, there are a cofinal subset $S$ of $\mu, \beta \in \mu$, and $m \in \omega$ so that $\beta_{x}=\beta$ and $m_{x}=m$ for all $x \in S$.

Set $A=\left\{k \in \omega:\left\langle f_{s}(k)\right\rangle_{s \in S}\right.$ is eventually bounded $\}$. By Lemma 2, $A$ is a finite set. Let $h$ be a natural number exceeding $\max (A)$. Set $R=(\beta, \mu) \cup\{\langle k, n\rangle$ : $k \geqslant h, m$ and $\left.f_{\beta}(k)<n\right\}$. Then $R$ is an open set, and since $\mu \backslash R$ is compact there is a finite subset $\mathscr{G}^{\prime}$ of $\mathscr{B}$ so that $\mu \backslash R \subset \cup \Re^{\prime}$. Let

$$
\Re=\mathscr{R}^{\prime} \cup\{R\} \cup\left\{\{x\}: x \notin \cup\left(\mathscr{R}^{\prime} \cup\{R\}\right)\right\} .
$$

Then $\mathscr{R}$ is a locally finite open cover of $X$. Let $M$ be a finite refiner of $\mathcal{R}$. By Lemma 1 it suffices to show that $M$ is a refiner of $\mathscr{B}$. We assume $M \subset R$. Set $M_{1}=M \cap \mu$ and set $M_{2}=M \cap(\omega \times \omega)$. List the members of $M_{2}$ as $\left\langle k_{1}, n_{1}\right\rangle,\left\langle k_{2}, n_{2}\right\rangle, \ldots$, $\left\langle k_{j}, n_{j}\right\rangle$, where $k_{1} \leqslant k_{2} \leqslant \cdots \leqslant k_{j}$. Let $q=\max \left\{n_{1}, n_{2}, \ldots, n_{j}\right\}$. There is an $s^{\prime} \in S$ so that $s^{\prime}>\max M_{1}$. Since $k_{1} \geqslant h, k_{1} \notin A$ so there is an $s \in S$ with $s>s^{\prime}$ so $f_{s}\left(k_{1}\right)>q$. Let $1 \leqslant d \leqslant j$. Since $M \subset R, f_{\beta}\left(k_{d}\right)<n_{d} \leqslant q<f_{s}\left(k_{1}\right) \leqslant f_{s}\left(k_{d}\right)$. Thus $M_{2} \subset\left\{\langle k, n\rangle: k \geqslant m, f_{\beta}(k)<n \leqslant f_{s}(k)\right\} \subset U(s, \beta, m)$. It follows that $M \subset$ $U(s, \beta, m)$.

$X$ is not almost $\boldsymbol{\aleph}_{0}$-fully normal. Set $\mathscr{B}^{\prime}=\{U(x, 0,0): x \in \mu\}$ and set $\mathscr{B}=\mathscr{B}^{\prime} \cup$ $\{\{x\}: x \in \omega \times \omega\}$. Suppose $\mathcal{L}$ is an open locally finite cover of $X$ with the property

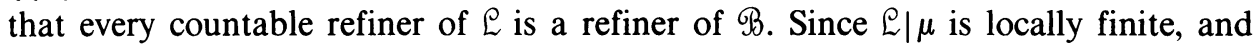
hence finite, only finitely many members of $\mathcal{L}$ meet $\mu$; list these members as $L_{1}$, $L_{2}, \ldots, L_{z}$. For each $x \in \mu$, there are $k_{x}, \beta_{x}$ and $n_{x}$ so that $x \in U\left(x, \beta_{x}, n_{x}\right) \subset L_{k_{x}}$, 
where $U\left(x, \beta_{x}, n_{x}\right)$ is a basic open set about $x$. By the Pressing-Down Lemma, there is a cofinal subset $\tilde{S}$ of $\mu, \tilde{m} \in \omega, \tilde{k}$ with $1 \leqslant \tilde{k} \leqslant z$, and $\tilde{\beta} \in \mu$ so that for each $x \in \tilde{S}, k_{x}=\tilde{k}, \beta_{x}=\tilde{\beta}$ and $n_{x}=\tilde{m}$. Set $\tilde{A}=\left\{k \in \omega:\left\langle f_{s}(k)\right\rangle_{s \in \tilde{S}}\right.$ is eventually bounded $\}$. Then $\tilde{A}$ is finite and there is a natural number $\tilde{h}$ exceeding $\max (\tilde{A})$. Set

$$
\tilde{R}=(\tilde{\beta}, \mu) \cup\left\{\langle k, n\rangle: k \geqslant \tilde{h}, \tilde{m} \text { and } f_{\tilde{\beta}}(k)<n\right\} .
$$

Then $\tilde{R} \subset L_{\tilde{k}}$ so each countable subset of $\tilde{R}$ is a refiner of $\mathscr{B}$. Let $D=\tilde{R} \backslash \mu$. Then $D$ is a refiner of $\mathscr{B}$ - a contradiction.

In his thesis [5, Theorem 2.2.10], H. J. K. Junnila proves that a space $X$ is 2-fully normal if and only if it is almost 2-fully normal and for each open cover $\mathcal{C}$ of $X$ there is a reflexive relation $V$ on $X$ such that, for each $x \in X, V(x)$ is open and such that,

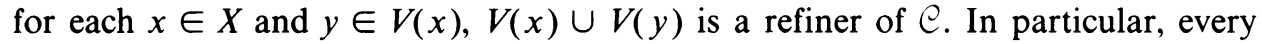
orthocompact almost 2-fully normal space is 2-fully normal. It is unknown whether the converse holds; the referee suggests that the space we have considered above could possibly be used in the construction of a counterexample.

Since the space $X$ was constructed as an example of a space that does not have a countably-compact-ification, it is interesting to note that nearly the same method of proof establishes that the following countably compact normal space $Y$ is not $q$-complete.

Let $\left\langle A_{\alpha}\right\rangle_{\alpha \in \mu}$ be an increasing maximal tower on $\omega$, where $\mu$ is a regular cardinal. Let $Y=\mu \cup \omega$ and, as usual, define a topology on $Y$ by specifying the following neighborhoods: Points of $\omega$ are isolated. If $0 \leqslant \beta<\alpha<\mu$ and $F$ is a finite subset of $\omega$, set

$$
U(\alpha, \beta, F)=(\beta, \alpha] \cup\left[A_{\alpha} \backslash A_{\beta}\right] \backslash F,
$$

and if $\alpha=0$ and $F$ is a finite subset of $\omega$, set

$$
U(0, \beta, F)=\{0\} \cup\left(A_{0} \backslash F\right) .
$$

Then $\mathscr{F}=\operatorname{fil}\left\{\left(\omega \backslash A_{\alpha}\right) \backslash F: \alpha \in \mu\right.$ and $F$ is a finite subset of $\left.\omega\right\}$ is a filter without a cluster point that is a Cauchy filter with respect to each quasi-uniformity that $Y$ admits.

The similarity of the methods of proof that $X$ and $Y$ are not $q$-complete is not just a coincidence. The basic' neighborhoods of points of $\mu$ in $X$ can be defined in terms of the following tower on $\omega \times \omega:\left\langle\left\{\langle k, n\rangle: k \in \omega, n \leqslant f_{\alpha}(k)\right\}\right\rangle_{\alpha \in \mu}$.

\section{REFERENCES}

1. G. Aquaro, Intorno ad una generalizzazione degli spazi paracompatti, Atti Accad. Naz. Lincei Rend. Cl. Sci. Fis. Mat. Natur. (8) 38 (1965), 824-827.

2. D. K. Burke and E. K. van Douwen, On countably compact extensions of normal locally compact M-spaces, Set-Theoretic Topology (Papers, Inst. Medicine and Math., Ohio Univ., Athens, Ohio, 1975-1976), Academic Press, New York, 1977, pp. 81-90.

3. P. Fletcher and W. F. Lindgren, Quasi-uniform spaces, Lecture Notes in Pure and Appl. Math., vol. 77. Dekker, New York, 1982.

4. K. P. Hart, More on M. E. Rudin's Dowker space, Proc. Amer. Math. Soc. 86 (1982), 508-510. 
5. H. J. K. Junnila, Covering properties and quasi-uniformities of topological spaces, $\mathrm{Ph}$. D. Thesis, Virginia Polytechnic Inst. and State Univ., Blacksburg, Va., 1978.

6. M. J. Mansfield, Some generalizations of full normality, Trans. Amer. Math. Soc. 86 (1957), 489-505.

7. K. Morita, Paracompactness and product spaces, Fund. Math. 50 (1961/1962), 223-236.

8. W. J. Pervin, Quasi-uniformization of topological spaces, Math. Ann. 147 (1962), 316-317.

Department of Mathematics, Universität Bern, CH-3012 Bern, Switzerland

Department of Mathematics, Virginia Polytech Institute \& State University, Blacksburg, VIRGINIA 24061 (Current address of both authors) 